\title{
ISOMETRIC RESULTS ON A MEASURE OF
}

\section{NON-COMPACTNESS FOR OPERATORS ON BANACH SPACES}

\author{
S.J. DILWORTH
}

\begin{abstract}
For each $\lambda \geq 1$ a class of Banach spaces $\phi_{\lambda}$ is defined.
Isometric results are obtained on the equivalence between a measure of non-compactness and the essential norm of a linear operator defined on a $\phi_{\lambda}$ space. Best values of $\lambda$ for the classical Banach spaces and for spaces with unconditional basis are investigated. For the space $c$ of convergent sequences the non-existence of a $\lambda$-unconditional basis with $\lambda<2$ is deduced.
\end{abstract}

Recall that a Banach space $E$ is said to be a $\pi_{\lambda}$ space $(1 \leq \lambda<\infty)$ if for every finite dimensional subspace $G$ of $E$ and for each $\epsilon>0$ there exist a finite-dimensional subspace $H \supseteq G$ and a projection $P$ from $E$ onto $H$ with ||$P|| \leq \lambda+\varepsilon$ (see [6]). We need the following dual notion.

DEFINITION 1. Suppose that $1 \leq \lambda<\infty$. A Banach space $E$ will be said to be a $\phi_{\lambda}$ space if for every closed subspace $M$ of finite codimension in $E$ and for each $\varepsilon>0$ there exist a closed subspace

Received 7 February 1986.

Copyright Clearance Centre, Inc. Serial-fee code: 0004-9727/87 $\$ A 2.00+0.00$. 
$N \subseteq M$ of finite codimension and a projection $P$ from $E$ onto $N$ with ||$P|| \leq \lambda+\varepsilon$.

The following proposition is essentially known (see [4]), but we indicate a short proof.

PROPOSITION 2. (a) If $E^{*}$ is a $\pi_{\lambda}$ space then $E$ is a $\phi_{1+\lambda}$ space.

(b) If $E$ is a $\phi_{\lambda}$ space then $E^{*}$ is a $\pi_{1+\lambda}$ space.

Proof. (a) Let $M$ be a closed subspace of finite codimension in $E$ and let $E>0$ be given. By a consequence of the principle of local reflexivity (see [6]) there exists a finite dimensional subspace $H$ containing $M_{\perp}=\left\{f \in E^{\star}: f(x)=0\right.$ for all $\left.x \in M\right\}$ and a weak*continuous projection $P$ from $E^{*}$ onto $H$ with $\|P\| \leq \lambda+\varepsilon$. Then $\left.\left(I-P^{\star}\right)\right|_{E}$ is a projection whose range is a subspace of finite codimension contained in $M$ and ||$I-P^{\star}|| \leq 1+\lambda+\varepsilon$. So $E$ is a $\phi_{1+\lambda}$ space.

(b) This is a simple duality argument and will be omitted:

Let $T: E \rightarrow F$ be a bounded operator between Banach spaces $E$ and $F$. The essential norm of $T$, denoted ||$T||_{e}$, is defined by $\|T\|_{e}=\inf \{\mid T+K \|: K: E \rightarrow F$ is a compact operator $\}$. Following [7] we define a measure of non-compactness of $T$, denoted $c(T)$, by $c(T)=\inf \left\{\left.|| T\right|_{M} \|: \operatorname{codim}(M)<\infty\right\}$. The familiar Kuratowski measure of non-compactness, $\gamma(T)$, which is defined by $\gamma(T)=\inf \{r$ : the image of the unit ball of $E$ is covered by finitely many balls in $F$ of radius $r\}$, is related to $c(T)$ by the inequalities $\frac{1}{2} c(T) \leq \gamma(T) \leq 2 c(T)$ (see [7]).

PROPOSITION 3. Suppose that $E$ is a $\phi_{\lambda}$ space and that $T: E \rightarrow F$ is a bounded operator. Then ||$T \|_{e} \leq \lambda c(T)$; in particular, ||$T||_{e} \leq \lambda|| T^{*} \mid \|_{e}$

Proof. Suppose that $K: E \rightarrow F$ is any compact operator and let $\varepsilon>0$ be given. Then $c(K)=0$ and so there exists a closed subspace $L$ of finite codimension such that $\left.|| K\right|_{L}||<\varepsilon$. Let $M$ be any closed subspace of finite codimension. Since $E$ is a $\phi_{\lambda}$ space there exists a closed subspace $N \subseteq L \cap M$ of finite codimension in $E$ and a 
projection $P$ from $E$ onto $N$ with $\|P\| \leq \lambda+\varepsilon$. Then $(T+K) P$ is a compact perturbation of $T$ and $\|(T+K) P\| \leq(\lambda+\varepsilon)\left(\left.|| T\right|_{M}||+\varepsilon\right)$. since $\varepsilon$ and $M$ are arbitrary it follows that ||$T||_{e} \leq \lambda c(T)$. We obviously have $c\left(T^{* *}\right) \leq|| T^{* *}\left\|_{e} \leq|| T^{*}\right\|_{e}$, while $c\left(T^{* *}\right) \geq c\left(T^{*}\right)$ follows easily from the definition of the measure of non-compactness $c(\cdot)$. Combining these inequalities gives ||$T||_{e} \leq \lambda|| T \star \|_{e}$.

Remark 4, It is not known whether there exists a constant $K$ such that $\|T\|_{e} \leq K|| T *||_{e}$ for all Banach spaces $E$ and $F$ and operators $T: E \rightarrow F$. The unpublished folklore result $a_{n}(T) \leq 3 a_{n}\left(T^{*}\right)$, where $a_{n}(T)$ denotes the $n^{\text {th }}$ approximation number of $T$, shows that $\left.|| T\right|_{e} \leq 3|| T^{\star}||_{e}$ provided $E^{\star}$ has the approximation property, and so the second statement in Proposition 3 is good only for $\lambda<3$.

COROLLARY 5. Suppose that $E$ is a classical Banach space and that $T: E \rightarrow F$ is a bounded operator. Then $\|T\|_{e} \leq 2 c(T)$ (and so ||$\left.T\left\|_{e} \leq 2|| T^{*} \mid\right\|_{e}\right)$

Proof. $E^{*}$ is a $\pi_{1}$-space, and so the result follows from Propositions 2 and 3.

Remark 6. The constant 2 is best possible (see Corollary 10(a) below). Results related to Proposition 3 are obtained in [7] under the assumption that $F$ has the compact approximation property but without any assumption on $E$. In [1] the Banach spaces for which $\gamma(T)$ and ||$T \|_{e}$ are equivalent semi-norms are characterized.

Now suppose that $E$ is a Banach space with a Schauder basis $\left(e_{k}\right)_{k=1}^{\infty}$. The basis constant $\mu$ is defined by $\mu=\sup \left\{|| P_{n}||: n \geq 1\right\}$, where $P_{n}$ is the natural projection from $E$ onto $\left[e_{k}\right]_{k=1}^{n}$ (the closed linear span of $\left.e_{1}, e_{2}, \ldots, e_{n}\right)$. The basis is said to be shrinking if ||$f \mid\left[e_{k}\right]_{k=\mathrm{n}}^{\infty} \| \rightarrow 0$ as $n \rightarrow \infty$ for every $f \in E^{*}$. Further, the basis is said to be $\lambda$-unconditional if $\left\|\sum_{1}^{n} \pm a_{k} e_{k}\right\| \leq \lambda\left\|\sum_{1}^{\infty} a_{k} e_{k}\right\|$ for all $n \geq 1$, for all scalars $\left(a_{k}\right)_{k=1}^{\infty}$, and for all choices of signs. It 
follows from Proposition 2 that if $E$ has a shrinking basis, with basis constant $\mu$, then $E$ is a $\phi_{1+\mu}$ space. We have the following refinement for spaces with a $\lambda$-unconditional shrinking basis.

PROPOSITION 7. Suppose that $E$ has a $\lambda$-unconditional shminking basis. Then $E$ is a $\phi_{\lambda}$ space.

Proof. For each $x=\sum_{1}^{\infty} a_{k} e_{k}$ in $E$ we define $\left\||| x|\|=\sup | \mid \sum_{1}^{\infty}\right.$ $\pm a_{k} e_{k}||$, where the supremum is taken over all choices of signs. Since ||$x|| \leq \||| x|||\leq \lambda||x| \mid$ it is sufficient to prove the proposition for the norm \|\|$\cdot\|\|$, for which $\left(e_{k}\right)_{k=1}^{\infty}$ is a 1 -unconditional basis; so we may assume that $\lambda=1$. Suppose that $\varepsilon>0$ and that $M$ is any closed subspace of codimension one in $E$. We prove the claim that there exists a subspace $N \subset M$ of finite codimension in $E$ which is $(1+\varepsilon)-$ complemented in $E$ and which possesses a $(1+\varepsilon)$-unconditional shrinking basis. There exists $f \in E^{*}$ such that $\|f\|=1$ and $M=\{x \in E: f(x)$ $=0\}$. We may choose $x \in E$ and a positive integer $n_{0}$ such that $f(x)=1,|| x|| \leq 2$, and $x=\sum_{k=1}^{n_{0}} x_{k} e_{k}$. Given $n>0$ there exists $n_{1}>n_{0}$ such that ||$f\left|\left[e_{k}\right]_{k=n_{1}}^{\infty}\right| \mid \leq n$. Then for any $m \geq n_{1}$ and for all scalars $a_{n_{1}}, \ldots, a_{m}$. we have

$$
\left(1-2 n l\left\||| \sum_{n_{1}}^{m} a_{k} e_{k}\right\| \leq \leq\left\||| \sum_{n_{1}}^{m} a_{k} f_{k}\right\| \leq(1+2 \eta)\left\||| \sum_{n_{1}}^{m} a_{k} e_{k}\right\| \|,\right.
$$

where $f_{k}=e_{k}-f\left(e_{k}\right) x$. Let $P$ be the natural projection from $E$ onto $\left[e_{k}\right]_{k=n_{1}}^{\infty}$ (which is a contraction because the basis is 1-unconditional); then $P$ is an isomorphism from $\left[f_{k}\right]_{k=n_{1}}^{\infty}$ onto $\left[\mathrm{e}_{\mathbf{k}}\right]_{\mathbf{k}=\mathrm{n}_{1}}^{\infty}$ with $\|P\||| p^{-1} \| \leq \frac{1+2 \eta}{1-2 \eta} \cdot$ Moreover,

$$
Q=\left(P \mid\left[f_{k}\right]_{k=n_{1}}^{\infty}\right)^{-1} \circ P
$$

is a projection from $E$ onto $\left[f_{k}\right]_{k=n_{1}}^{\infty}$ with ||$Q|| \leq \frac{1+2 n}{1-2 n}$. The claim 
now follows by taking $\eta$ sufficiently small. The general result for a closed subspace $M$ of arbitrary finite codimension is obatined by applying the claim finitely many times and by considering a subspace of codimension one at each stage of the argument.

Remark 8. Say that a schauder basis $\left(e_{k}\right)_{k=1}^{\infty}$ is $\lambda$-bimonotone if $\sup \left\{|| P_{n}||,\left\|I-P_{n}\right\|: n \geq 1\right\} \leq \lambda$. Then the proof of Proposition 7 shows that $E$ is a $\phi_{\lambda}$ space if $\left(e_{k}\right)_{k=1}^{\infty}$ is a $\lambda$-bimonotone shrinking basis of $E$.

Let $c$ denote the space of convergent sequences $x=\left(x_{k}\right)_{k=1}^{\infty}$ with the norm ||$x||=\sup \left|x_{k}\right|$, and let $c_{0}$ be the subspace of sequences which tend to zero; let $\ell p \quad(1 \leq p<\infty)$ denote the space of sequences for which

$$
\|x\|_{p}=\left(\sum_{1}^{\infty}\left|x_{k}\right|^{p}\right)^{1 / p}<\infty
$$

COROLLARY 9. The Banach spaces $c_{0}$ and $\operatorname{lp}(1<p<\infty)$ are $\phi_{1}$ spaces.

COROLLARY 10. (a) $c$ and $\ell_{1}$ are $\phi_{2}$ spaces but are not $\phi_{\lambda}$ spaces for any $\lambda<2$.

(b) Let $\left(e_{k}\right)_{k=1}^{\infty}$ be a $\lambda$-unconditional basis for $c$. Then $\lambda \geq 2$; in particular, the Banach-Mazur distance from $c$ to any space with a 1-unconditional basis is at least 2.

Proof. (a) Let $I: \ell_{1} \rightarrow c_{0}$ be the formal identity operator and let $j: c_{0} \rightarrow c$ be the natural inclusion. Let $\left(e_{k}\right)_{k=1}^{\infty}$ be the standard basis of $\ell_{1}$ and define $K: \ell_{1} \rightarrow c$ by $K\left(e_{k}\right)=u(k \geq 1)$, where $u$ is the sequence which has every term equal to one. Then ||$j I-\frac{1}{2} K||=\frac{1}{2}$, and so $c(I) \leq \frac{1}{2}$; it now follows from Proposition 3 that $l_{1}$ is not a $\phi_{\lambda}$ space for any $\lambda<2$. Let $M$ be any subspace of finite codimension contained in $c_{0}$ and let $P$ be a projection on $c$ whose range is $M$. 
Then $P\left(I-\frac{1}{2} K\right)$ is a compact perturbation of $I$, and so ||$P\left(j I-\frac{1}{2} K\right)|| \geq 1$. It follows that ||$P|| \geq 2$, and so $c$ is not a $\phi_{\lambda}$ space for any $\lambda<2$. The fact that $c$ and $\ell_{1}$ are $\phi_{2}$ spaces is a consequence of Proposition 2 .

(b) Any unconditional basis of $c_{0}$ (and hence of $c$ ) is equivalent to the standard basis (see for example [8, p.71]), and so must be shrinking. The result now follows from (a) and proposition 7.

Remark 11. C.V. Hutton ([5]) discussed the formal identity from $\ell_{1}$ to $c_{0}$ as an example of an operator $T$ with the property that $a_{n}(T) \neq a_{n}\left(T^{*}\right)$

Remark 12. Banach $\left(\left[2\right.\right.$, p.242]) asked whether $c$ and $c_{0}$ were almost isometric. Cambern proved in [3] that the Banach-Mazur distance from $c$ to $c_{0}$ is 3 .

It is very easy to prove, in fact, that it is not possible to imbed an infinite-dimensional $C(K)$ space almost isometrically into $c_{0}$, as the following lemra shows.

LEMMA 13. Let $K$ be an infinite compact Hausdorff space and let $T: C(K) \rightarrow c_{0}$ be a Banach isomorphism onto a subspace of $c_{0}$. Then $\|T\||| T^{-1}|| \geq 2$.

Proof. We may assume that ||$T||=1$; let $\left(e_{k}^{*}\right)_{k=1}^{\infty}$ denote the functionals biorthogonal to the standard basis of $c_{0}$. Given $\varepsilon>0$ there exists $n_{0}$ such that $\left|e_{k} T(1)\right|<\varepsilon$ for all $k>n_{0}$, where $1 \in C(K)$ is the constant one function. Select $y \in C(K)$ such that ||$y||=1$ and $e_{k}^{*}(T y)=0$ for $1 \leq k \leq n_{0}$. Then $\max (\|x+y\|$, $\|x-y\| \mid=2$, whereas $\max (|| T x+T y\|\| T x-,T y \|) \leq 1+\varepsilon$, and it follows that ||$T^{-1} \| \geq 2$.

Remark 14. Say that $E$ has the distortion property (see [9]) if, given $\varepsilon>0$, a Banach space $F$ will contain a $(1+\varepsilon)$-isomorphic copy of $E$ whenever $E$ and $F$ are isomorphic. It is well known that $c_{0}$ 
and $l_{1}$ share this property, but since $c$ and $c_{0}$ are isomorphic the previous lemma shows (taking $C(K)=c)$ that $c$ coes not.

\section{References}

[1] K. Astala and H.-O. Tylli, "On bounded compactness property and measures of non-compactness", J. Funct. Anal. (to appear).

[2] S. Banach, Theorie des Operations Linearies (Second Edition), Chelsea Publishing Company, New York, 1978.

[3] M. Cambern, "On mappings of sequence spaces", Studia Math. 30 (1968), 73-77.

[4] W.J. Davis, "Remarks on finite rank projections", J. Approx. Theory 9 (1973), 205-211.

[5] C.V. Hutton, "On the approximation numbers of an operator and its adjoint", Math. Ann. 210 (1974), 277-280.

[6] W.B. Johnson, H.P. Rosenthal and M. Zippin, "On bases, finite dimensional decompositions and weaker structures in Banach spaces", Israel J. Math. 9 (1971), 488-506.

[1] A. Lebow and M. Schechter, "Semigroups of operators and measure of non-compactness", J. Fronct. Anal. 7 (1971), 1-26.

[8] J. Lindenstrauss and L. Tzafriri, Classical Banach spaces $I$, Springer-Verlag, 1977.

[q] J. Lindenstrauss and A. Pelczyrski, "Contributions to the theory of the classical Banach spaces, J. Funct. Anal. 8 (1971), 225-249.

Department of Mathematics,

The University of Texas at Austin,

Austin,

Texas 78712

United States of America. 